\title{
Complications of midurethral slings and their management
}

\author{
Victor W. Nitti, MD \\ Professor and Vice Chairman, Department of Urology, and Director of Female Pelvic Medicine and Reconstructive Surgery, New York University Langone Medical Center, New York, NY
}

Cite as: Can Urol Assoc J 2012;6(5):S120-2. htrp://dx.doi.org/10.5489/cuaj.12197

\section{Abstract}

While midurethral slings are associated with a low rate of morbidity, complications can arise intraoperatively or following the procedure. Management of midurethral sling complications is relatively straightforward and urgency is dictated by the severity of the complications, potential for long-term negative effects, and bother to the patient.

M idurethral synthetic slings are currently the most common surgical treatment for women with stress urinary incontinence (SUI). While generally well tolerated and safe, these operations are not free of complications. This review will discuss the more common complications that can be associated with these interventions and provide some guidance on how to deal with them if they arise.

\section{Intraoperative complications}

Potential intraoperative complications include bleeding, urethral and bladder injury. The overall relative risk of perioperative complications for transobturator procedures has been compared to retropubic procedures in a meta-analysis. ${ }^{1}$ The overall risk of complications related to surgery for SUI is considerably lower with the transobturator approach (pooled odds ratio 0.40 in randomized trials and 0.21 in observational cohort studies; Fig. 1).

\section{Significant bleeding}

Significant bleeding is a potential intraoperative complication of midurethral sling insertion, occurring in less than $1 \%$ of procedures. Additionally, evidence suggests that the likelihood is lower with the transobturator approach relative to retropubic slings., ${ }^{2,3}$

If bleeding is encountered, one should attempt to control the vessel if possible. Otherwise, the best advice is to complete the procedure as quickly as possible. There are many different approaches that can be used to manage the bleeding itself, including tamponade, hemostatic agents, vaginal packing, and embolization.

\section{Bladder and urethral injuries}

Using the retropubic approach, there is an approximate 5\% risk of bladder or urethral perforation with trocar passage. The risk is considerably lower $(<1 \%)$ with the transobturator approach. Expert recommendations from the American Urological Association (AUA) state that intraoperative cystoscopy should always be performed, to help minimize the risk of urinary tract injury. ${ }^{1}$

Should a bladder injury occur, the trocar should be removed and replaced. Depending on the size of the injury, one might consider draining the bladder for 24 to 48 hours with an indwelling catheter. Most bladder injuries during SUI surgery are inconsequential, with no effect on outcomes.

Urethral injury usually results from dissection in the wrong plane. Urethral repair should be done using 4-0 Vicryl, and the periurethral fascia should be closed. The American Urological Association's (AUA) recommendation is that "synthetic sling surgery is contraindicated in stress incontinent patients with a concurrent urethrovaginal fistula, urethral erosion, intraoperative urethral injury and/or urethral diverticulum."4

Other, less common potential intraoperative complications include bowel injury (retropubic slings) and nerve injury.

\section{Early postoperative complications}

Early postoperative complications include lower urinary tract symptoms (LUTS) and voiding dysfunction, infection, extrusion and pain.

\section{LUTS and voiding dysfunction}

Initial urgency and urgency incontinence can occur in up to $20 \%$ of patients following insertion of a midurethral sling. In a metaanalysis of randomized trials, there was no significant difference in the development of these symptoms between the transobturator and retropubic approaches. ${ }^{1}$

To ensure that the patient is emptying, post-void residual should be checked. Infection should also be ruled out.

Should the problem(s) persist, the initial management should be behavioural. One can consider initiating an anticholinergic agent, 
but waiting to judge the impact of behavioural modification is a reasonable approach. Note that these symptoms may resolve without treatment.

\section{Pain}

Pain should be expected to some degree. Possible causes include hematoma, positioning, retention, and nerve entrapment. When using the transobturator approach, there may also be pain due to trauma to the adductor longus.

Pain should be treated expectantly for the first one to two weeks after surgery. If it persists beyond this period, one should then consider the possibility of nerve or muscle injury. This can be treated with pain medication, but if it remains problematic, consider imaging and possible sling excision.

\section{Late postoperative complications}

These are often the most challenging to treat and include extrusion (vaginal exposure), erosion (mesh inside lower urinary or gastrointestinal tract), obstruction/voiding dysfunction and recurrent urinary tract infections.

\section{Extrusion}

Recent data indicate that extrusion rates are in the range of $1.1 \%$ (TVT-O) to $2.6 \%$ (TVT). It may occur without symptoms, or may be associated with bleeding, discharge, discomfort, pain, dyspareunia or hispareunia.

Management of extrusion can range from very conservative (i.e., observation) to a more aggressive approach. Surgical options include excision of the extruded segment (which is the preferred method), burying of the extruded segment or removal of all graft materials (e.g., if infected or recurrent). In case of surgical intervention, it is not our practice to place another synthetic sling at the same time.

\section{Erosion}

Erosion of slings into the urethra is quite uncommon $(<1 \%)$. Although sling material found in the bladder or urethra is often attributed to "erosion" in many cases it occurs as a result of a missed perforation.

Management of a bladder erosion can be done endoscopically, laparoscopically or openly through a retropubic or vaginal approach. For urethral erosions and erosions into the bladder that involve a large surface area, open excision may be preferred over endoscopic excision. Laparoscopy-assisted endoscopic excision is another option; it may be preferred for an intravesical sling placement. As with extrusions, the placement of another sling at the same time as the excision of an erosion is generally not recommended.

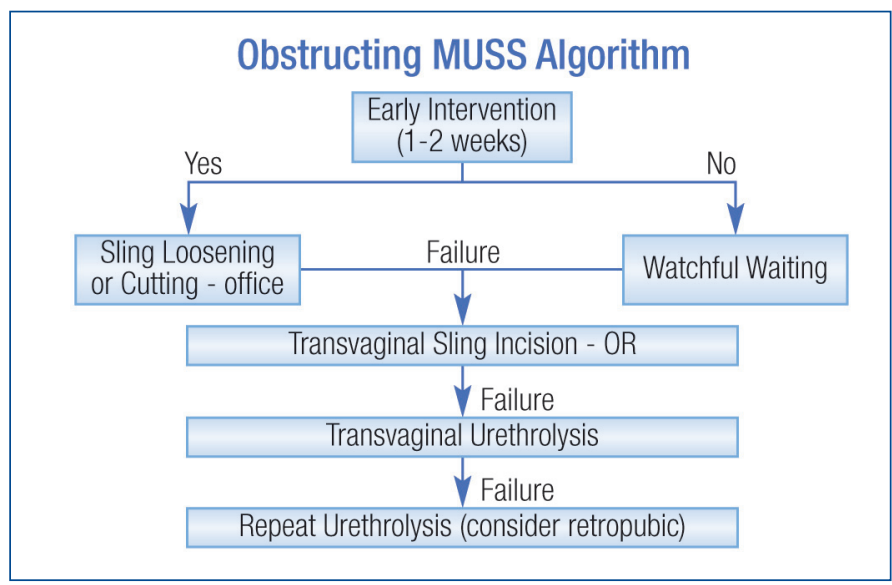

Fig. 1. Algorithm for management of obstructive symptoms associated with midurethral slings.

\section{Obstruction/voiding dysfunction}

The true incidence of obstruction and voiding dysfunction after incontinence surgery is not known. However, data from most large series indicate that the intervention rate for these complications following midurethral slings is between $1 \%$ and $2 \%$.

Obstructive symptoms include urinary retention or incomplete emptying, obstructive voiding symptoms and storage symptoms (e.g., frequency, urgency, urge incontinence). Nonobstructive symptoms include storage symptoms, with or without detrusor overactivity.

Evaluation includes a physical examination looking for "over correction," hypermobility, cystocele, enterocele, rectocele or uterine prolapse. History should include preoperative voiding and continence status, and the timing of symptom onset. The number and type of procedures should also be documented. In many cases, the physical examination and history may be sufficient to guide management. Other cases may require endoscopy and/or urodynamics.

Early intervention is indicated for women in retention requiring catheterization. Beyond that, the timing of intervention will depend on the degree of bother. The only absolute selection criterion for surgical intervention is a temporal relationship between surgery and onset of voiding symptoms.

For nonobstructive problems causing mainly storage symptoms, options include behavioural modification, antimuscarinics, and neuromodulation and botulinum toxin. Definitive treatment options for obstruction include sling loosening, sling loosening incision and urethrolysis (Fig. 1). In the case of a midurethral synthetic sling it is imperative to identify the sling and cut or loosen it.

Competing interests: Dr. Nitti is an ongoing paid consultant with Allergan, Astellas, Coloplast, Medtronic, Serenity Pharmaceuticals, and Uroplasty. 


\section{References}

1. Sung VW, Schleinitz MD, Rardin CR, et al. Comparison of retropubic vs transobturator approach to midurethral slings: a systematic review and meta-analysis. Am J Obstet Gynecol 2007;197:3-11. http:// dx.doi.org/10.1016/i.ajog.2006.09.025

2. Agostini $A$, Bretelle F, Franchi F, et al. Immediate complications of tension-free vaginal tape (TVT): results of a French survey. Eur J Obstet Gynecol Reprod Biol 2006;124:237-9. http://dx.doi.org/10.1016/i. ejogrb.2005.06.035

3. Kölle D, Tamussino K, Hanzal E, et al. Bleeding complications with the tensionfree vaginal tape operation. Am J Obstet Gynecol 2005;193:2045-9. hittp://dx.doi.org/10.1016/i.ajog.2005.06.061
4. Dmochowski RR, Blaivas JM, Gormley EA, et al. Update of AUA guideline on the surgical management of female stress urinary incontinence. J Urol 2010;183:1906-14. http://dx.doi.org/10.1016/i. juro.2010.02.2369

5. Latthe PM, Singh P, Foon R, et al. Two routes of transobturator tape procedures in stress urinary incontinence: a meta-analysis with direct and indirect comparison of randomized trials. BJU Int 2010;106:68-76. http://dx.doi.org/10.1111/j.1464-410X.2009.09051.x

Correspondence: Dr. Victor Nitti, NYU Urology Associates, 150 East 32nd St., 2nd Floor, New York, NY 10016; victor.nitti@nyumc.org 